\title{
Adaptive Mixed Finite Element Method for Elliptic Problems with Concentrated Source Terms
}

\author{
Muhammad llyas ${ }^{1}$, Agah D. Garnadi ${ }^{2}$, Sri Nurdiati \\ ${ }^{1}$ Mathematics, Faculty of Science and Information Technology, The University of Newcastle, \\ Callaghan 2308, Australia \\ ${ }^{2}$ Mathematics, Faculty of Mathematics and Natural Sciences, Bogor Agricultural University, \\ Bogor 16680, Indonesia \\ Correspondence: E-mail: muhammad.ilyas@uon.edu.au
}

\section{A B S T RACT}

An adaptive mixed finite element method using the Lagrange multiplier technique is used to solve elliptic problems with delta Dirac source terms. The problem arises in the use of Chow-Anderssen linear functional methodology to recover coefficients locally in parameter estimation of an elliptic equation from a point-wise measurement. In this article, we used a posterior error estimator based on averaging technique as refinement indicators to produce a cycle of mesh adaptation, which is experimentally shown to capture singularity phenomena. Our numerical results showed that the adaptive refinement process successfully refines elements around the center of the source terms. The results also showed that the global error estimation is better than uniform refinement process in terms of computation time.

\section{ARTICLE INFO}

Article History: Submitted/Received 20 Oct 2018 First revised 20 Des 2018 Accepted 20 Feb 2019 First available online $22 \mathrm{Feb} 2019$ Publication date 01 Sep 2019

\section{Keywords:}

Adaptive,

Mixed finite element method,

Posteriori error estimates,

Point source function. 


\section{INTRODUCTION}

In the study of the steady-state flow of groundwater in a confined aquifer $\Omega$, the governing equation is the following elliptic partial differential equation (Yeh, 1986).

$-\nabla \cdot(\alpha \nabla u)=\mathbf{f}$ in $\Omega$,

where $\Omega \subset \square^{d}, d \in\{1,2,3\}$ is a bounded domain, $\quad \alpha: \Omega \rightarrow \square$ denotes the transmissivity coefficient, $u: \Omega \rightarrow \square$ is pressure and $f$ is the source term. When the source term $f$ and the coefficient $\alpha$ are given, appropriate boundary information about the solution $u$ is required so that the corresponding forward (direct) problem of determining $u$ in $\Omega$ has a unique solution. Conversely, the recovery of information about the coefficient $\alpha$, when $u$ and $\mathbf{f}$ are given, is an inverse problem with $\alpha$ being sensitive to perturbations in $u$ and $\mathbf{f}$. It is the recovery of $\alpha$ from measurements of $u$ and $f$, through the utilization of equation (1), that is called the aquifer parameter identification problem.

Previous study (Zwolak, 2008) examined the numerical performance of ansatz for the recovery of aquifer transmissivity from observational data. A particular problem of our interest is in the case of $\mathbf{f}$ is a point source function. To this end, we solve a Poisson problem with deltaDirac right hand side function and homogenous Dirichlet boundary condition:

$\left\{\begin{array}{cl}-\Delta u=\delta_{x_{0}} & \text { in } \quad \Omega, \\ u=0 & \text { on } \quad \partial \Omega,\end{array}\right.$

where $\Omega \subset \square^{2}$ is a bounded polygonal domain and $\delta_{x_{0}}$ is delta-Dirac function at point $x_{0}$ inside region $\Omega$. Note that problem (2) arises in diverse fields such as the electric field generated by point charge, transport equations for efficient discharge in aquatic media, etc.

Due to the singular characteristics of the solution of the problem (2), mesh refinements around the source point $x_{0}$ are required to improve the quality of the approximation. In this paper, we used a posteriori error control based on the averaging technique for the Poisson problem (Bahriawati and Carstensen, 2005). Furthermore, we restrict our study for two-dimensional case, but this technique can be extended to a threedimensional case by selecting a corresponding adaptive method.

\section{ADAPTIVE METHOD}

Let $T$ be a quasi-uniform partition of the domain $\Omega$. In simplication, we applied the adaptive procedure similar to literature (Adler et al., 2011) as follows: We solve the problem on the mesh to obtain a solution of the mixed finite element method (SOLVE). A posteriori error estimates the global error $\eta$ and local errors associated with each element $\eta_{K}, K \quad T \in \quad$ (ESTIMATE).

We mark the element based on marking strategy for refinement (MARK ). The procedure ended with mesh refinement using bisection method of the marked element (REFINE). In short, adaptive finite element methods typically consist of successive loops of the sequence:

$$
\text { SOLVE } \rightarrow \text { ESTIMATE } \rightarrow \text { MARK } \rightarrow \text { REFINE }
$$

\subsection{Solve and Estimate}

In this paper, we use the mixed finite element method with Lagrange multiplier technique based on (Bahriawati and Carstensen, 2005) to obtain flux $p_{h}$ and 
solution $u_{h}$. The a posteriori error estimates global error $\eta$ and local errors for each element $\eta_{K}$ as follows $\eta^{2}=\sum_{K \in T} \eta_{K}^{2}$.

\subsection{Mark}

Then we compare global error $\eta$ and error tolerance $\varepsilon$. If $\eta \leq \varepsilon$, then we are done since the required tolerance has been achieved, otherwise we select subset $M \subset T$ which contains the elements where the local errors are relatively large. In our problem, we expect that elements close to $x_{0}$ to be marked for refinement since this area is where the error will be the most significant. We ordered elements based on their local error and then selecting few first of them to construct a set $M$ satisfies the following criteria: given a marking parameter $\vartheta$, where $0<\theta<1$, find a subset $M \subset T$ such that $\sum_{K \in M} \eta_{K}^{2} \geq \theta \eta^{2}$.

\subsection{Refine}

Mesh refinement is done using the vertex bisection method (Adler et al., 2011). This method chooses a vertex of each marked element $K \in T$ as the peak of the element and creates an additional node at the edge opposite (known as the base of $K)$. The bisection is done by connecting the peak node to the additional node. If the adjacent element $K$, is not refined, then our additional node will be a hanging node. Therefore, we also need to refine the element $K$ which shares the same base with $K$. This type of refinement is chosen assuming that the support of regularised delta Dirac function is equally of the same distance from the triangle edges and the inclusion triangle always understood of an isosceles triangle. Other cases need careful analysis related to the error estimator such as for CR-basis (ShaoHong and XiaoPing, 2008).

\section{NUMERICAL EXPERIMENTAL RESULTS AND DISCUSSION}

We have done a numerical experiment to assess the performance of an adaptive mesh-refinement strategy based on a posteriori error control. To simulate a delta Dirac function, we define $f_{x_{0}}(x)=\frac{1}{\left|B\left(x_{0}, a\right)\right|} \chi_{B\left(x_{0}, a\right)}(x), \quad$ where $B(x 0, a)$ is the ball centered at $x_{0}$ with radius $a$ and $\chi_{B\left(x_{0}, a\right)}$ is the characteristic function of the ball $B\left(x_{0} a\right)$. As we progress $a \rightarrow 0$, this function will behave similar to a delta Dirac function (Tiandho, 2017).

We initiate the process with a very coarse mesh. At each step, our refinement method creates a new mesh better adapted to the solution of problem (2). To view a concentrated force function $f_{x_{0}}$ on point $x_{0}$, we initialize $a$ longer than the edge of element containing the point $x_{0}$. After several iterations, we set smaller values of $a$ and continue the process.

The problem is solved in the square $\Omega=(-1,1) \times(-1,1)$, with $x_{0}=(0,0)$. We choose Dirichlet boundary conditions and initialise $a=0.4$ and then set new values for $a$ to $0.2 ; 0.1 ; 0.05$ consecutively after three iterations. Then, we set $a=0.01$ until error tolerance reached, which we set $\varepsilon=0.01$. For this problem, we set our refinement parameter to be $\theta=0.6$, which means we adaptively refine $60 \%$ of the domain. Figure 1 shows mesh grid and displacement for our initial iteration.

Figures 2, 3, 4, 5, and 6 show some of the successive both refined meshes and displacements created in the process guided by $\eta$. We demonstrated the first iteration of different values of $a$, where $a \in[0.2,0.1,0.05,0.01]$ with number of repetition and the number of degree of 
freedom (d.o.f.) of each respective mesh and displacement prescribed on the caption of each figure.

From these figures, we can see that our method successfully refine the mesh grid around $x_{0}$. The progression of a simulates the delta Dirac function as expected. We can see that the mesh grid creates a "ring" around $x_{0}$ that slowly grows closer to the centre because of our choices of approximation function. Figure 6 shows the final iteration when the global error

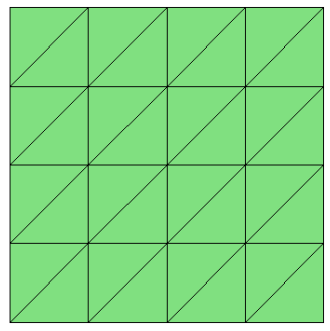

(a) Mesh grid is just below the error tolerance and as we can see that the mesh around $x_{0}$ is finely refined.

Figure 7 shows the error curve for our adaptive method compared to uniform refinement process. Using our adaptive approach, the low global error is achieved in a far smaller number of elements than uniform refinement, meaning that our adaptive method is better than uniform refinement in terms of computational cost.

Figure 1. $a=0.4, \theta=0.6$, iter $=1$, d.o.f. $=32$.

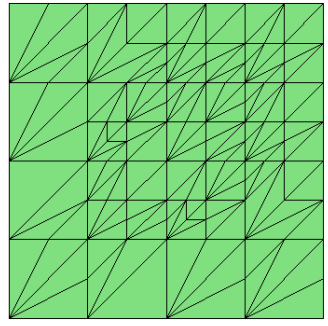

(a) Mesh grid

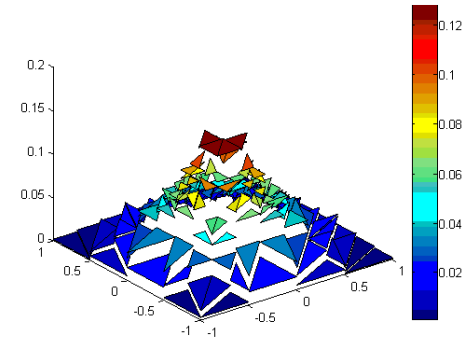

(b) Displacement

Figure 2. $a=0.2, \theta=0.6$, iter $=4$, d.o.f. $=162$. 


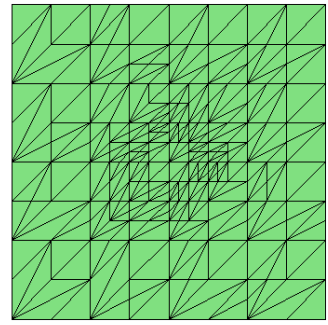

(a) Mesh grid

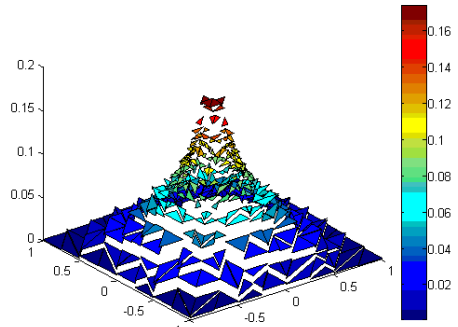

(b) Displacement

Figure 3. $a=0.1, \theta=0.6$, iter $=7$, d.o.f. $=354$.

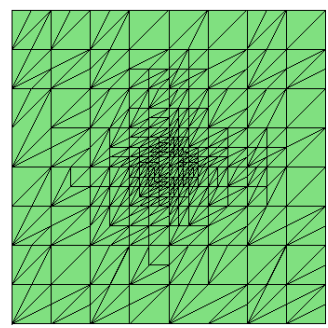

(a) Mesh grid

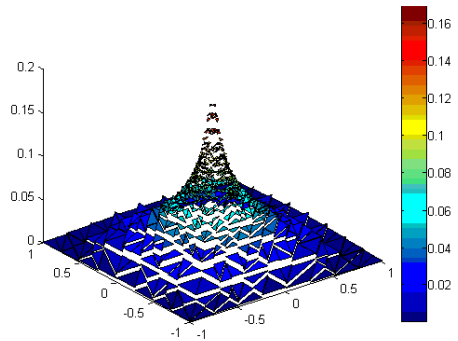

(b) Displacement

Figure 4. $a=0.05, \theta=0.6$, iter $=10$, d.o.f. $=706$.

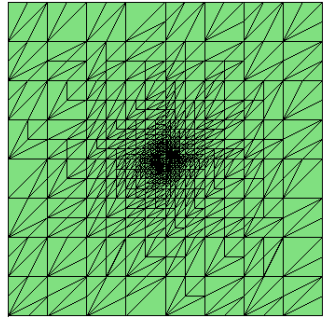

(a) Mesh grid

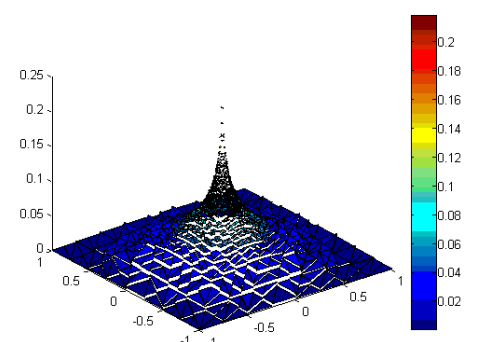

(b) Displacement

Figure 5. $a=0.01, \theta=0.6$, iter $=13$, d.o.f. $=1457$.

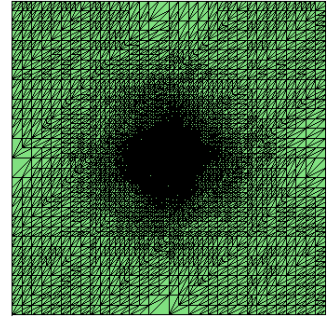

(a) Mesh grid

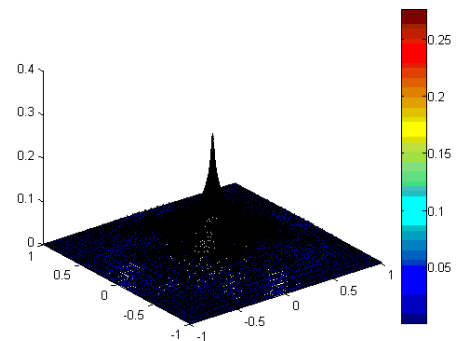

(b) Displacement

Figure 6. $a=0.01, \theta=0.6$, iter $=24$, d.o.f. $=26790$. 


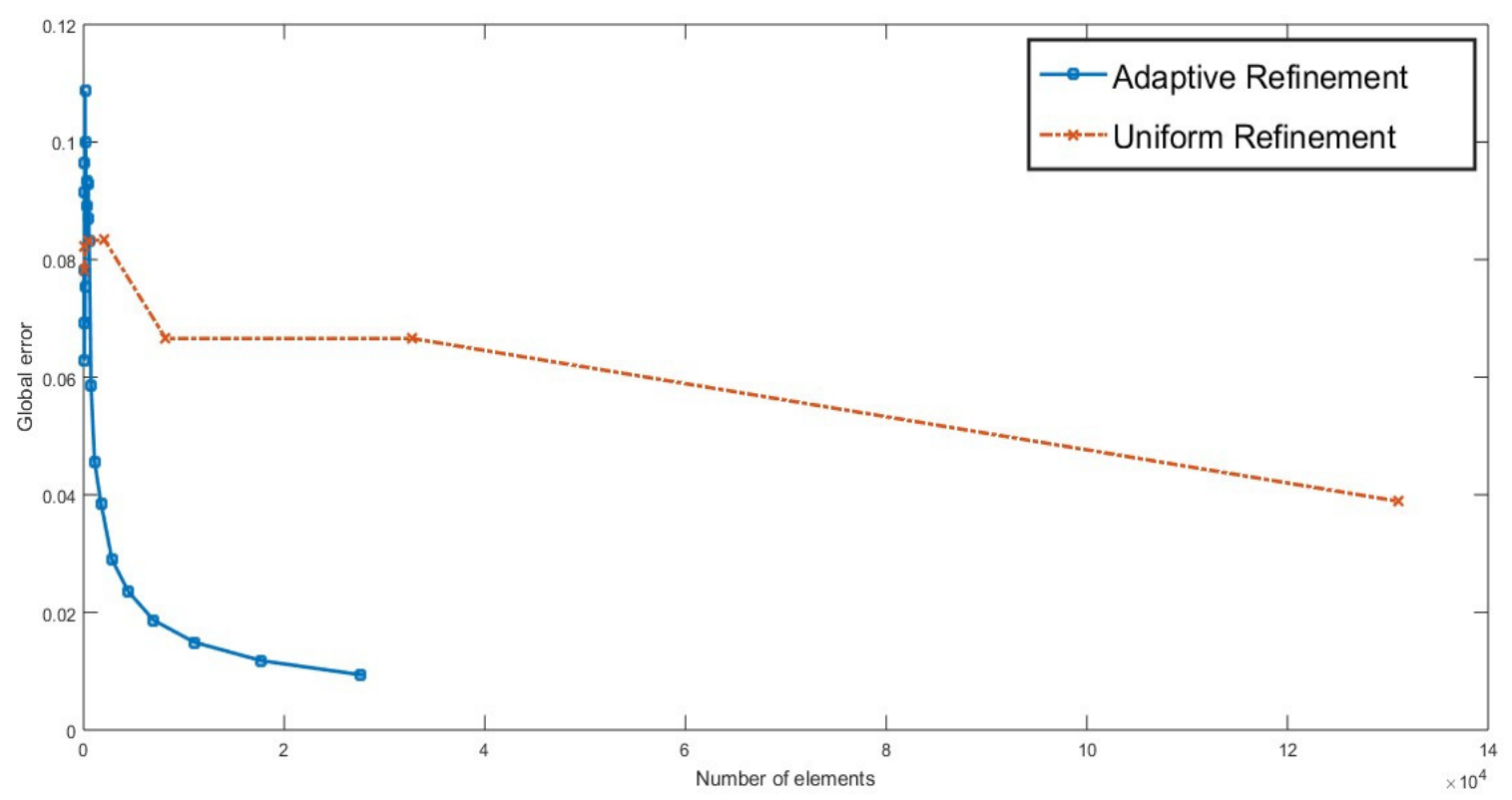

Figure 7. Error curves for adaptive and uniform conditions.

\section{CONCLUSION}

We have applied a posteriori error control to estimate mixed finite element approximation for the Poisson problem with delta Dirac function source terms. An adaptive algorithm is introduced to refine elements guided by the error estimator. Our result shows that the adaptive refinement process is successfully improved elements around the centre of the source terms. We also show that the global error estimation is better than uniform refinement process. Furthermore, we could extend our adaptive refinement method for other problems and by some modifications for threedimensional case.

\section{ACKNOWLEDGEMENTS}

We acknowledged the Government of Indonesia, The Ministry of Research, Technology and Higher Education, through 'PUPT-IPB' research grant, under contract: 083/SP2H/PL/Dit.Litabmas/II/2015.

\section{AUTHORS' NOTE}

The author(s) declare(s) that there is no conflict of interest regarding the publication of this article. Authors confirmed that the data and the paper are free of plagiarism.

\section{REFERENCES}

Adler, J.H., Manteuffel, T.A., McCormick, S.F., Nolting, J.W., Ruge, J.W., and Tang, L. (2011). Efficiency based adaptive local refinement for first-order system least-squares formulations. SIAM Journal on Scientific Computing, 33(1), 1-24.

Bahriawati, C. and Carstensen, C. (2005). Three MATLAB implementations of the lowest-order Raviart-Thomas MFEM with a posteriori error control. Computational Methods in Applied Mathematics Computer Methods Applied Mathematics, 5(4), 333-361. 
269 | Indonesian Journal of Science \& Technology, Volume 4 Issue 2, September 2019 page 263-269

ShaoHong , D., and XiaoPing ,X. (2008). Residual -based a posteriori error estimates of nonconforming finite element method for elliptic problems with Dirac delta source terms . Science in China Series A: Mathematics, 51(8), 1440-1460.

Tiandho, Y. (2017) Dirac particles emission from an elliptical black hole. Indonesian Journal of Science and Technology, 2(1), 50-56.

Yeh, W. (1986). Review of parameter-identification procedures in groundwater hydrology - the inverse problem. Water Research Research, 22, 95-108.

Zwolak, M. (2008). Numerical ansatz for solving integro-differential equations with increasingly smooth memory kernels: spin-boson model and beyond. Computational Science and Discovery, 1(1), 1-19. 